\title{
Colecalciferol: Uma análise crítica de produtos manipulados e industrializados
}

\author{
Colecalciferol: A critical analysis of manipulated and \\ industrialized products
}

Recebido em: 19/07/2018 Aceito em: $21 / 11 / 2018$
Stephanye Carolyne Christino CHAGAS ${ }^{1}$; Maria Amélia Paiva FERRUCCI'; Julia Celly de Morais CARVALHO'; ${ }^{1}$ Asley Thalia Medeiros SOUZA ${ }^{1}$; Davi Pereira de SANTANA ${ }^{1,2}$; Leila Bastos LEAL ${ }^{1,2}$

${ }^{1}$ Núcleo de Desenvolvimento Farmacêutico e Cosmético (NUDFAC), Universidade Federal de Pernambuco - UFPE. Av. Professor Artur de Sá, CEP 50740-521. Recife, PE, Brasil. ${ }^{2}$ Farmácia Escola Carlos Drummond de Andrade (FECDA) e Universidade Federal de Pernambuco-UFPE. Av. Professor Artur de Sá, CEP 50740-521. Recife, PE, Brasil. E-mail: carolchagas.90@gmail.com

\section{ABSTRACT}

Vitamin D deficiency is a worldwide problem, and according to several studies, reports indicate an increase in the number of prescriptions containing vitamin D by Health workers. Also, individuals have used this substance for another purpose other than those linked to mineral metabolism, despite there is no self-evidence for the use of vitamin D in preventing other diseases. This work has critically analysed the prescribing, manipulation and use of cholecalciferol in extemporaneous (compound) preparations and commercially available products. Compound pharmacies informed the types of oral dosage forms containing cholecalciferol in Recife, Brazil; Electronic queries to the Anvisa website and INTUITIVE ${ }^{\circledR}$ (software used by the Carlos Drummond de Andrade Pharmacy School-FECDA) allowed the commercial and compounded formulations to be listed and analysed. Databases were evaluated to establish a critical and current analysis of our results. There was widespread concern about the establishment of adequate blood levels of this vitamin in the population by health professionals since 29 different specialists prescribed cholecalciferol in extemporaneous formulations. A higher amount of dosage and pharmaceutical products were also found when compared to the marketed national products. Moreover, ensuring that these prescriptions are based on the need of each patient requires blood tests and the use of recognized guidelines, which, combined with pharmacotherapeutic monitoring, may contribute to therapeutic success.

Keywords: vitamin D; cholecalciferol; therapy; safety.

\section{RESUMO}

Profissionais de saúde tem prescrito cada vez mais vitamina D, visto que sua deficiência representa um problema de saúde coletiva mundial. Adicionalmente, indivíduos têm utilizado esta substância para outros fins além daqueles ligados ao metabolismo mineral, apesar da escassez de dados científicos sobre seu papel na prevenção de outras doenças. Este trabalho analisou a prescrição, manipulação e uso do colecalciferol em apresentações magistrais e comerciais nacionais. Farmácias magistrais de Recife, PE informaram as for- 
mas farmacêuticas manipuladas contendo colecalciferol para administração oral; consultas eletrônicas ao site da Anvisa e ao software INTUITIVE®, utilizado pela Farmácia Escola Carlos Drummond de Andrade (FECDA), permitiram que as apresentações comerciais/manipuladas, no período do estudo, fossem elencadas e analisadas. Foram realizadas consultas a bases de dados visando estabelecer uma análise crítica e atual diante dos achados. Foi verificada uma ampla preocupação no estabelecimento dos níveis sanguíneos adequados desta vitamina na população por parte dos profissionais de saúde habilitados, visto que 29 diferentes especialistas prescreveram colecalciferol manipulado. Também foi constatada uma maior quantidade de dosagens e formas farmacêuticas magistrais quando comparadas com as apresentações comerciais do mercado nacional. No mais, garantir que estas prescrições estejam embasadas nas necessidades de cada paciente, requer exames sanguíneos e utilização de guias reconhecidos, o que, associado ao acompanhamento farmacoterapêutico, poderá contribuir para o sucesso terapêutico.

Palavras-chave: vitamina D; colecalciferol; terapia; segurança.

\section{INTRODUÇÃO}

Vitamina D é um termo geral utilizado para representar um grupo de hormônios esteroides com diferentes graus de atividade (1). Apesar da existência de diferentes formas químicas desta vitamina, o colecalciferol (vitamina D3) e ergocalciferol (vitamina D2) revelam maior interesse prático e científico (2).

No ser humano, a obtenção desta vitamina pode ocorrer a partir da exposição solar, da dieta e, quando necessário, da suplementação $(2,3)$. A exposição da pele aos raios do sol apresenta-se como a principal fonte da vitamina D3, que é produzida por meio de uma reação fotolítica, não enzimática, mediada pelos raios ultravioleta B (UVB) que convertem o 7-diidrocolesterol presente na epiderme, em pré-vitamina $\mathrm{D}_{3}$. Em seguida, ocorre uma isomerização térmica direcionando a formação da vitamina $D_{3}$, que é transportada para o fígado, por meio da proteína ligadora de vitamina D, onde é hidroxilada pelas 25-hidroxilases hepáticas mitocondriais e microssomais, dando origem à 25 -hidroxivitamina $\mathrm{D}(25(\mathrm{OH}) \mathrm{D})$ com tempo de meia vida entre 21 e 30 dias (4-6). A produção de $25(\mathrm{OH}) \mathrm{D}$ no fígado é rápida e sofre pouca regulação, sendo esta a forma de vitamina D circulante predominante e seus níveis séricos refletem os níveis de reserva corporal desta vitamina. Assim, a sua quantificação é a forma mais indicada na avaliação do seu status corporal, uma vez que se relaciona não apenas com a síntese cutânea, mas também com a ingestão $(7,8)$.
Fontes endógenas de vitamina D permanecem duas vezes mais tempo no organismo que as exógenas. Entretanto, a vitamina D oriunda da exposição solar é influenciada por diversos fatores, como estação do ano, pigmentação da pele e uso de protetor solar, e a sua ingestão natural através da dieta, representa apenas uma pequena parte necessária para atender as necessidades humanas (5). Desta forma, na falta de uma exposição regular à luz do sol, apenas as fontes alimentares não são suficientes para manter níveis adequados e por isto a suplementação com medicamentos tem sido constantemente necessária (9).

A quantidade suplementar que deve ser administrada diariamente tem sido objeto de consideráveis debates no século XXI. Atualmente, o Institute of Medicine (IOM) recomenda uma dose que pode variar de 800 a 4000 UI por dia, para doenças relacionadas ao metabolismo mineral, a depender da idade do paciente (10). No entanto, a suplementação com vitamina $\mathrm{D}$ tem sido realizada em maiores doses e com maior frequência devido a achados associando seus baixos níveis sanguíneos com diversas doenças crônicas, apesar da falta de dados científicos consistentes sobre o papel desta molécula na prevenção de doenças que não as do metabolismo mineral $(11,12)$. Há a probabilidade de ocorrer intoxicação quando doses superiores a 10.000 UI de Vitamina D2 ou D3/dia são administradas por vários meses a anos (13). Assim, uma dose tóxica de Vitamina D mediante a suplementação é uma possibilidade real, haja vista, inclusive, que vários relatos de caso têm sido publicados, associando efeitos colaterais 
tóxicos vinculados a altas dosagens de vitamina D (14-18). Adicionalmente, outros relatos de caso envolvem a intoxicação por colecalciferol em decorrência de erros na manipulação $(9,19,20)$. Casos como estes, destoam das Boas Práticas de Manipulação (BPM) devidamente regulamentadas pela Agência Nacional de Vigilância Sanitária (Anvisa) por meio das Resoluções RDC 67/2007 (21) e RDC 87/2008 (22).

A dispensação de medicamentos na farmácia magistral é de particular importância, visto que é possível manipular ativos em diferentes dosagens, associações e formas farmacêuticas, a depender da prescrição (23). Considerando que mais da metade dos medicamentos prescritos, distribuídos ou vendidos em todo o mundo são inadequados e que a prescrição inadequada pode levar a eventos adversos evitáveis, o farmacêutico deve atuar cada vez mais no acompanhamento farmacoterapêtico do paciente, auxiliando-o na promoção do uso seguro e ra- cional de medicamentos, para além do âmbito hospitalar como é mais habitual (24).

Assim, esse estudo teve como objetivo analisar de forma crítica a prescrição, manipulação e uso de colecalciferol a partir de apresentações magistrais da Farmácia Escola Carlos Drummond de Andrade (FECDA), localizada em Recife, PE, e de apresentações comerciais produzidas pelas indústrias de medicamentos brasileiras.

\section{MÉTODOS}

Relação e tratamento dos dados dos medicamentos industriais contendo colecalciferol. Por meio do serviço online disponibilizado pela Anvisa, foi realizada consulta e relacionados todos os medicamentos contendo colecalciferol para administração oral, associados ou não, que possuíam registro ativo neste órgão fiscalizador. A Figura 1 detalha a execução da pesquisa bem como os critérios de classificação considerados.

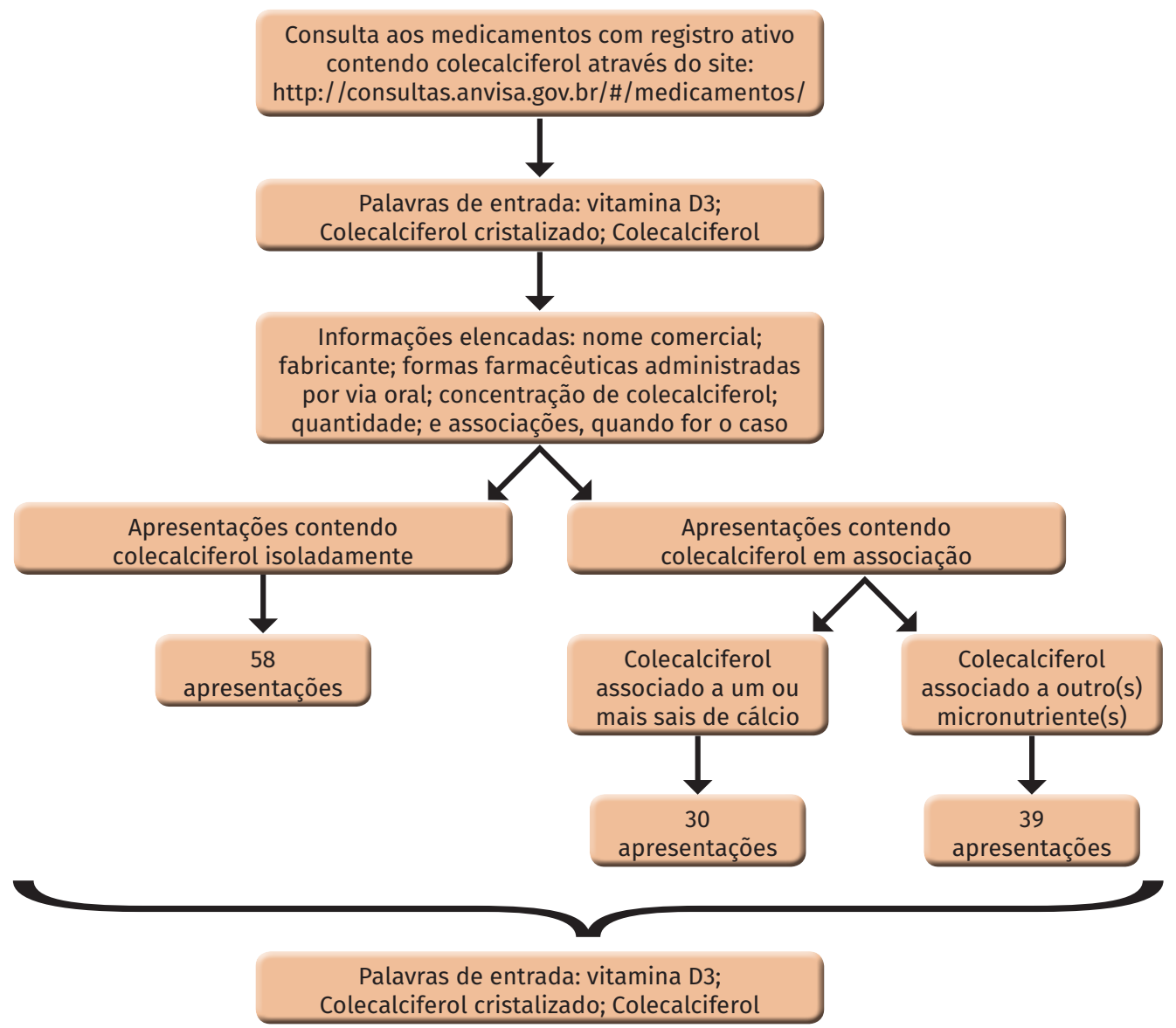

Figura 1 - Metodologia aplicada para avaliação dos medicamentos contendo colecalciferol disponíveis no mercado nacional 
Relação e tratamento dos dados das formas farmacêuticas magistrais. Todas as redes de farmácias magistrais localizadas em Recife, PE, forneceram informações relativas às formas farmacêuticas manipuladas contendo apenas colecalciferol para administração oral em atendimento às prescrições dos profissionais de saúde habilitados. Diante das informações, uma análise crítica foi realizada em relação à biodisponibilidade destas preparações e vantagens/ desvantagens daí decorrentes, foram discutidas através de embasamento científico apropriado.

Relação e tratamento dos dados das formulações magistrais contendo colecalciferol. Formulações contendo colecalciferol, na forma farmacêutica cápsula dura oral, manipuladas pela FECDA, foram catalogadas no período entre outubro e dezembro de 2016. As informações foram classificadas seguindo os critérios detalhados na Figura 2, e os dados foram tratados utilizando o software Microsoft Excel®.

Análise crítica dos dados. Após a obtenção dos dados dos medicamentos industrializados, das diferentes formas farmacêuticas magistrais manipuladas em farmácias magistrais de Recife-PE e do perfil das formulações magistrais dispensadas no último trimestre de 2016 pela FECDA, foi possível realizar uma análise crítica e integrada com a literatura atual, a respeito da forma farmacêutica, concentração, posologia, perfil de prescritores e uso seguro e racional. Foram utilizados os seguintes bancos de dados: science direct, pubmed, google acadêmico e scielo, empregando como entrada, em conjunto ou disjunção, as palavras: colecalciferol, vitamina D, revisão, hipervitaminose $\mathrm{D}$, intoxicação, segurança, guias, mercado.

A pesquisa bibliográfica foi realizada entre fevereiro e abril de 2017, sendo selecionadas apenas as informações que tinham interesse para os objetivos propostos. Os critérios de inclusão usados foram dissertações, livros, artigos originais e de revisão, que abordassem a temática em estudo, publicados, entre 2002 e 2016, na língua portuguesa, inglesa, francesa ou espanhola. Por outro lado, foram excluídos artigos e revisões bibliográficas ou sistemáticas sobre outras vitaminas exceto a vitamina $\mathrm{D}$ e/ou que abordassem esta vitamina em outros contextos que não os estabelecidos nos objetivos propostos.

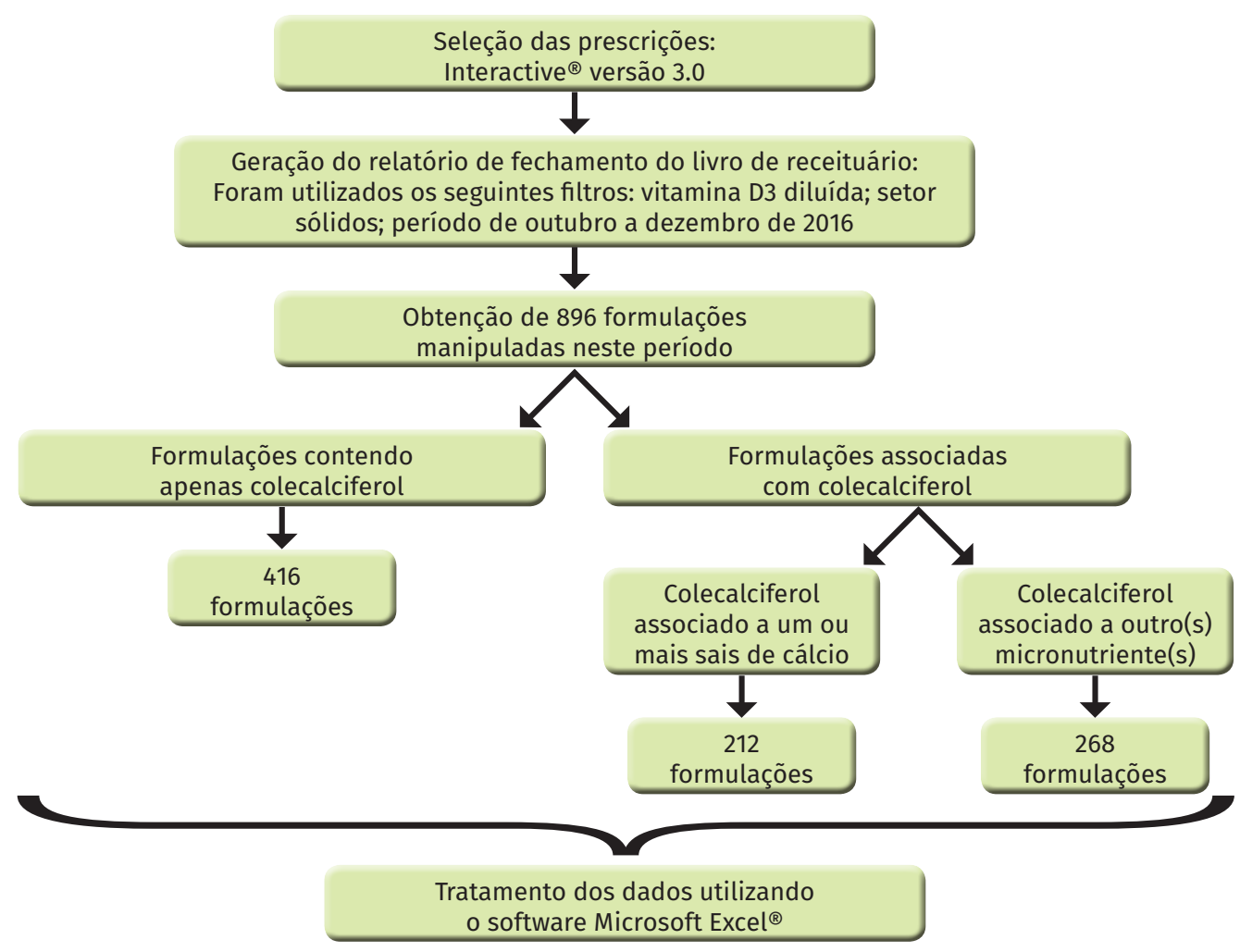

Figura 2. Metodologia aplicada para geração do perfil de prescrições de cápsulas duras orais contendo colecalciferol. 


\section{RESULTADOS E DISCUSSÃO}

O Quadro 1 apresenta todas as formas farmacêuticas contendo apenas colecalciferol fabricadas pelas indústrias farmacêuticas, com registro ativo no mercado nacional e as formas farmacêuticas manipuladas em Recife, PE, contendo o mesmo fármaco, isoladamente, para administração oral. Uma das razões de tamanha variedade de formas farmacêuticas apresentadas pela farmácia magistral reside no preparo em caráter extemporâneo, viabilizando a veiculação de diversos fármacos em formas farmacêuticas onde estes poderiam apresentar estabilidade limitada (23).

Mais que uma seleção baseada em condições específicas do paciente como idade, estado patológico ou sensibilidade, a escolha por uma determinada forma farmacêutica impactará diretamente na biodisponibilidade do ativo e, portanto, no seu regime terapêutico $(25,26)$. Deste modo, o tipo de forma farmacêutica oral influenciará na velocidade e/ou extensão de absorção no trato gastrointestinal.

Quadro 1. Formas farmacêuticas (via oral) fabricadas pela indústria brasileira contendo colecalciferol isoladamente em comparação com as formas farmacêuticas manipuladas e/ou fracionadas por farmácias magistrais de Recife, PE, Brasil.

\begin{tabular}{|l|c|c|}
\hline Forma farmacêutica & Industrial & Magistral \\
\hline Comprimido revestido & $\mathrm{X}$ & \\
\hline Cápsula dura oral & & $\mathrm{X}$ \\
\hline Cápsula dura oleosa & & $\mathrm{X}$ \\
\hline Cápsula mole oleosa & $\mathrm{X}$ & $\mathrm{X}^{*}$ \\
\hline Pastilha sublingual & & $\mathrm{X}$ \\
\hline Sachê & & $\mathrm{X}$ \\
\hline Gomas & & $\mathrm{X}$ \\
\hline Solução oral & $\mathrm{X}$ & $\mathrm{X}$ \\
\hline Xarope & & $\mathrm{X}$ \\
\hline
\end{tabular}

*fracionada

Para além da biodisponibilidade, em detrimento da forma farmacêutica, os regimes terapêuticos para tratamento com colecalciferol variam de acordo com o grau de deficiência desta substância, bem como da meta a ser atingida em termos de concentração sanguínea (27). A quantificação sérica de 25(OH)D3 é útil na avaliação do nível corporal de vitamina $\mathrm{D}$, sendo, portanto, uma resposta às fontes de obtenção. São estes dados que darão suporte ao prescritor para estabelecimento de um regime terapêutico racional caso haja a necessidade de uma suplementação $(5,28)$.

$\mathrm{Na}$ Tabela 1 estão expostas todas as apresentações comerciais contendo colecalciferol isoladamente. Ainda, existem 23 apresentações contendo colecalciferol associado a um ou mais sais de cálcio com registro ativo no Brasil, nas formas comprimido simples, revestido, efervescente e mastigável; solução e suspensão oral, além do pó para suspensão oral. No que concerne a apresentações contendo colecalciferol associado a outras vitaminas e minerais com registro ativo no Brasil, são em número de 31 , nas formas farmacêuticas comprimido simples, revestido, efervescente e mastigável; drágea e cápsula gelatinosa mole; xarope, solução, suspensão e emulsão oral.

Considerando apenas as formas farmacêuticas sólidas industrializadas, dentre as apresentações contendo colecalciferol isoladamente encontradas no mercado brasileiro, os prescritores habilitados podem estabelecer regimes posológicos baseados em apenas sete diferentes concentrações, sendo elas: 800 UI; 1.000 UI; 2.000 UI; 5.000 UI; 7.000 UI; 10.000 UI e 50.000 UI. O número de possíveis concentrações para atender às necessidades específicas do paciente baseadas em seu correto diagnóstico, varia amplamente no setor magistral, sendo esta uma de suas principais vantagens em relação aos medicamentos industrializados (23). A exemplo, dentre as 896 prescrições magistrais avaliadas contendo colecalciferol na forma farmacêutica cápsula dura oral manipulada na FECDA, no período do estudo, 416 continham colecalciferol isoladamente em 26 diferentes concentrações conforme apresentado na Figura 3.

No entanto, apesar da grande faixa de concentrações manipuladas e dispensadas no período citado, ao considerar a posologia e o período mínimo de 30 dias, muitas delas convergiam para mesma dose ou mesma quantidade total administrada ao final de um mês, como pode ser verificado nas setas de ligações mostradas na Figura 3. Considerando que a farmacocinética da vitamina D3 após a administração oral é de extrema relevância no estabelecimento 
de regimes posológicos adequados, estudos previamente realizados comparando à biodisponibilidade deste fármaco em resposta a dosagem diária e semanal ao longo de até 15 semanas, verificaram que não existe diferença relevante para a prática clínica (27, 29-31). Assim, um esquema de suplementação adequado deve ser estabelecido baseado na concentração sérica de vitamina D3 e buscando uma maior adesão do paciente à terapia. Um estudo conduzido por Remondi e cols (2012), apontou que em usuários de medicamentos contínuos, como é o caso do colecalciferol, a frequência de administração do(s) medicamento(s) ao longo do dia foi determinante na adesão à terapia, superando inclusive outros parâmetros como a complexidade global do regime, número de medicamentos, forma farmacêutica e ações para utilização do produto (32).

É possível encontrar na literatura, vários guias orientando a prescrição de vitamina D baseada na concentração sérica do paciente, entre eles: Vitamin D Clinical Guideline, da Clinical
Commissioning Group 2013 (33); Guideline: Vitamin D supplementation in pregnant women, da World Health Organization, 2012 (34); Evaluation, Treatment, and Prevention of Vitamin D Deficiency: An Endocrine Society Clinical Practice Guideline, da Endocrine Society 2011(35). Zittermann e cols (2014) estabeleceram uma fórmula para calcular os regimes de dose de vitamina $\mathrm{D}$, considerando o peso corporal, a idade, as concentrações iniciais de $25(\mathrm{OH}) \mathrm{D}$ em circulação e o tipo de suplemento de vitamina D (D2 ou D3) (36). Em concordância, a Sociedade Brasileira de Endocrinologia e Metabologia (SBEM), publicou um consenso em 2014 estabelecendo que, de uma maneira geral, quando a $25(\mathrm{OH}) \mathrm{D}$ está muito abaixo do desejado $(<20 \mathrm{ng} / \mathrm{mL})$, um esquema de ataque é necessário para repor os estoques corporais. O esquema mais utilizado atualmente é de $50.000 \mathrm{UI} / \mathrm{semana}$ (ou $7.000 \mathrm{UI} /$ dia) de vitamina D por 6 a 8 semanas (27). Caso a meta de $25(\mathrm{OH}) \mathrm{D}$ não tenha sido atingida, um novo ciclo pode ser proposto.

Tabela 1. Apresentações comerciais contendo colecalciferol, isoladamente e em associação, com registro ativo (Anvisa) no mercado brasileiro

\begin{tabular}{|c|c|c|c|}
\hline Nome comercial & Forma Farmacêutica & Concentração de colecalciferol & Quantidades (mL ou unidade)* \\
\hline \multirow{12}{*}{ Addera D3 } & \multirow{2}{*}{ Solução oral } & $3.300 \mathrm{UI} / \mathrm{mL}$ & $10 ; 15 ; 20 ; 30$ \\
\hline & & $10.000 \mathrm{UI} / \mathrm{mL}$ & 10 \\
\hline & \multirow{5}{*}{ Comprimido revestido } & $1.000 \mathrm{UI}$ & \multirow[t]{2}{*}{$10 ; 30$} \\
\hline & & $5.000 \mathrm{UI}$ & \\
\hline & & $7.000 \mathrm{UI}$ & \multirow{3}{*}{$4 ; 10 ; 30$} \\
\hline & & $10.000 \mathrm{UI}$ & \\
\hline & & $50.000 \mathrm{UI}$ & \\
\hline & \multirow{5}{*}{ Cápsula gelatinosa mole } & $1.000 \mathrm{UI}$ & \multirow[t]{2}{*}{$10 ; 30$} \\
\hline & & $5.000 \mathrm{UI}$ & \\
\hline & & $7.000 \mathrm{UI}$ & \multirow{3}{*}{$4 ; 10 ; 30$} \\
\hline & & $10.000 \mathrm{UI}$ & \\
\hline & & $50.000 \mathrm{UI}$ & \\
\hline \multirow{6}{*}{ AltaD } & \multirow{6}{*}{ Comprimido revestido } & $1.000 \mathrm{UI}$ & \multirow{2}{*}{$30 ; 60$} \\
\hline & & $2.000 \mathrm{UI}$ & \\
\hline & & $5.000 \mathrm{UI}$ & $8 ; 30$ \\
\hline & & $7.000 \mathrm{UI}$ & $4 ; 8 ; 30$ \\
\hline & & $10.000 \mathrm{UI}$ & \\
\hline & & $50.000 \mathrm{UI}$ & 4 \\
\hline
\end{tabular}




\begin{tabular}{|c|c|c|c|}
\hline Nome comercial & Forma Farmacêutica & Concentração de colecalciferol & Quantidades (mL ou unidade)* \\
\hline \multirow{7}{*}{ Depura } & Solução oral & $14.000 \mathrm{UI} / \mathrm{mL}$ & 10 \\
\hline & \multirow{6}{*}{ Comprimido revestido } & $1.000 \mathrm{UI}$ & \\
\hline & & $2.000 \mathrm{UI}$ & $30 ; 60$ \\
\hline & & $5.000 \mathrm{UI}$ & $8 ; 30$ \\
\hline & & $7.000 \mathrm{UI}$ & $4 ; 8 ; 30$ \\
\hline & & $10.000 \mathrm{UI}$ & 4 \\
\hline & & $50.000 \mathrm{UI}$ & 4 \\
\hline Degen & Cápsula gelatinosa mole & $1.000 \mathrm{UI}$ & $30 ; 60 ; 90 ; 180$ \\
\hline Doss & Cápsula gelatinosa mole & $1.000 \mathrm{UI}$ & $30 ; 60 ; 90 ; 180$ \\
\hline \multirow{6}{*}{ Dprev } & \multirow{6}{*}{ Comprimido revestido } & $1.000 \mathrm{UI}$ & $30 ; 60$ \\
\hline & & $2.000 \mathrm{UI}$ & \\
\hline & & $5.000 \mathrm{UI}$ & $8 ; 30$ \\
\hline & & $7.000 \mathrm{UI}$ & $4 ; 8 ; 30$ \\
\hline & & $10.000 \mathrm{UI}$ & 4 \\
\hline & & $50.000 \mathrm{UI}$ & 4 \\
\hline \multirow{12}{*}{ Refont D } & \multirow[t]{3}{*}{ Solução oral } & $3.300 \mathrm{UI} / \mathrm{mL}$ & $10 ; 15 ; 20 ; 30$ \\
\hline & & $1.000 \mathrm{UI}$ & $10 ; 30$ \\
\hline & & $5.000 \mathrm{UI}$ & \multirow{4}{*}{$4 ; 10 ; 30$} \\
\hline & \multirow[t]{3}{*}{ Comprimido revestido } & $7.000 \mathrm{UI}$ & \\
\hline & & $10.000 \mathrm{UI}$ & \\
\hline & & $50.000 \mathrm{UI}$ & \\
\hline & \multirow{5}{*}{ Cápsula gelatinosa mole } & $1.000 \mathrm{UI}$ & $10 ; 30$ \\
\hline & & $5.000 \mathrm{UI}$ & \multirow{4}{*}{$4 ; 10 ; 30$} \\
\hline & & $7.000 \mathrm{UI}$ & \\
\hline & & $10.000 \mathrm{UI}$ & \\
\hline & & $50.000 \mathrm{UI}$ & \\
\hline & Solução oral & $10.000 \mathrm{UI} / \mathrm{mL}$ & 10 \\
\hline \multirow{6}{*}{ OHDE } & \multirow{6}{*}{ Comprimido revestido } & $1.000 \mathrm{UI}$ & $30 ; 60$ \\
\hline & & $2.000 \mathrm{UI}$ & $30 ; 60$ \\
\hline & & $5.000 \mathrm{UI}$ & $8 ; 30$ \\
\hline & & $7.000 \mathrm{UI}$ & $4 ; 8 ; 30$ \\
\hline & & $10.000 \mathrm{UI}$ & \\
\hline & & $50.000 \mathrm{UI}$ & 4 \\
\hline \multirow{6}{*}{ Sany D } & \multirow{6}{*}{ Comprimido revestido } & $1.000 \mathrm{UI}$ & \multirow{2}{*}{$30 ; 60$} \\
\hline & & $2.000 \mathrm{UI}$ & \\
\hline & & $5.000 \mathrm{UI}$ & \multirow{2}{*}{$8 ; 30$} \\
\hline & & $7.000 \mathrm{UI}$ & \\
\hline & & $10.000 \mathrm{UI}$ & \multirow{2}{*}{4} \\
\hline & & $50.000 \mathrm{UI}$ & \\
\hline SanyD 800 & Cápsula gelatinosa mole & $800 \mathrm{UI}$ & $16 ; 30 ; 60$ \\
\hline
\end{tabular}

Como pode existir uma variação individual na resposta ao tratamento, devido a fatores como gestação, doença renal crônica, obesidade e pós-cirurgia bariátrica, uma reavaliação dos valores plasmáticos após cada ciclo se faz necessário. Após esse período, a dose de manutenção deve ser ins- tituída e varia de acordo com a faixa etária e com as condições concomitantes, que por sua vez, está relacionada a especialidade do prescritor (27).

Neste estudo, foi observado que os regimes terapêuticos contendo colecalciferol tanto isoladamente quanto em associação, foram prescritos por 
quase 30 diferentes especialidades médicas, como pode ser verificado nas Figuras 4 e 5 .

Os 15\% englobam 21 diferentes especialidades, incluindo geriatra. A razão para tamanha distribuição das prescrições em diferentes especialidades médicas pode ser justificada pela vitamina D está relacionada a uma série de vias metabólicas somado ao fato da hipovitaminose D representar hoje um problema de saúde coletiva mundial gerando cada vez mais preocupação dos profissionais em suprir sua deficiência $(1,13)$.

Os 15\% englobam 21 diferentes especialidades, incluindo geriatra. A razão para tamanha distribuição das prescrições em diferentes especialidades médicas pode ser justificada pela vitamina D está relacionada a uma série de vias metabólicas somado ao fato da hipovitaminose D representar hoje um problema de saúde coletiva mundial gerando cada vez mais preocupação dos profissionais em suprir sua deficiência $(1,13)$.

Atualmente, muitos brasileiros têm utilizado vitamina D3 como suplemento vitamínico para prevenção de diversas doenças crônicas, apesar da falta de dados científicos consistentes sobre o papel desta molécula na prevenção de doenças que não as do metabolismo mineral (17). Embora suplementos vitamínicos sejam muitas vezes usados com a in- tenção de alcançar benefícios para a saúde, efeitos cumulativos de seu uso generalizado, sem a correta indicação terapêutica, pode elevar os níveis séricos a concentrações acima do recomendado ocasionando efeitos adversos e intoxicação (37).

A intoxicação por vitamina $\mathrm{D}$ é muitas vezes de difícil detecção quando considerados apenas os sintomas clínicos que são não-específicos, entre eles: anorexia, vômitos, constipação, cefaléia, hipertensão, polidipsia e poliúria, dificultando inclusive a execução da farmacovigilância por parte do farmacêutico e outros profissionais de saúde $(17,38)$. Assim, investigações bioquímicas são necessárias. Além da concentração de $25(\mathrm{OH}) \mathrm{D}$, as concentrações de creatinina, cálcio e paratormônio (PTH) também devem ser investigadas (38). O IOM, estabeleceu como altas as concentrações de 25(OH)D entre 100 e $150 \mathrm{ng} / \mathrm{mL}$ e valores acima de $150 \mathrm{ng} / \mathrm{mL}$ foram considerados tóxico (10).

A hipervitaminose D aumenta a absorção intestinal de cálcio causando hipercalcemia, que, por sua vez, pode causar diversos efeitos colaterais, principalmente neurológicos, gastrointestinais e renais. A hipercalcemia aguda pode levar à lesão renal por vasoconstricção direta e pela redução no volume do líquido extracelular, devido aos sintomas clínicos relatados, como a anorexia, náuseas, vômitos e redução na capacidade de concentrar a urina.

\begin{tabular}{|c|c|c|c|}
\hline Concentração (UI) & Posologia & Concentração (UI) & Posologia \\
\hline 400 & 1/dia & 12.000 & 1/dia \\
\hline 800 & 1/dia & 13.000 & 1/dia \\
\hline 1.000 & 1/dia & 14.000 & $1 /$ semana \\
\hline 1.200 & 1/dia & 15.000 & $1 /$ semana \\
\hline 2.000 & 1/dia & 18.000 & 1/dia \\
\hline 2.500 & 1/dia & & 1/dia \\
\hline 3.000 & 1/dia & 20.000 & $1 /$ semana \\
\hline 3.500 & 1/dia & & 2/semana \\
\hline 4.000 & 1/dia & 21.000 & 1/semana \\
\hline \multirow{2}{*}{5.000} & 1/dia & 24.000 & $1 /$ semana \\
\hline & $2 /$ semana & \multirow{2}{*}{25.000} & $1 /$ semana \\
\hline 6.000 & 1/dia & & 2/semana \\
\hline \multirow{4}{*}{7.000} & 1/dia & 28.000 & 1/semana \\
\hline & $1 /$ semana & \multirow{2}{*}{30.000} & $1 /$ semana \\
\hline & $2 /$ semana & & 3/semana \\
\hline & 4/semana & \multirow{2}{*}{50.000} & 1/semana \\
\hline 8.000 & 2/dia & & $2 /$ semana \\
\hline \multirow{2}{*}{10.000} & 1/dia & & \\
\hline & $1 /$ semana & & \\
\hline
\end{tabular}

Figura 3. Concentrações de colecalciferol isoladamente manipulados na forma farmacêutica cápsula dura oral na FECDA no último trimestre de 2016. 


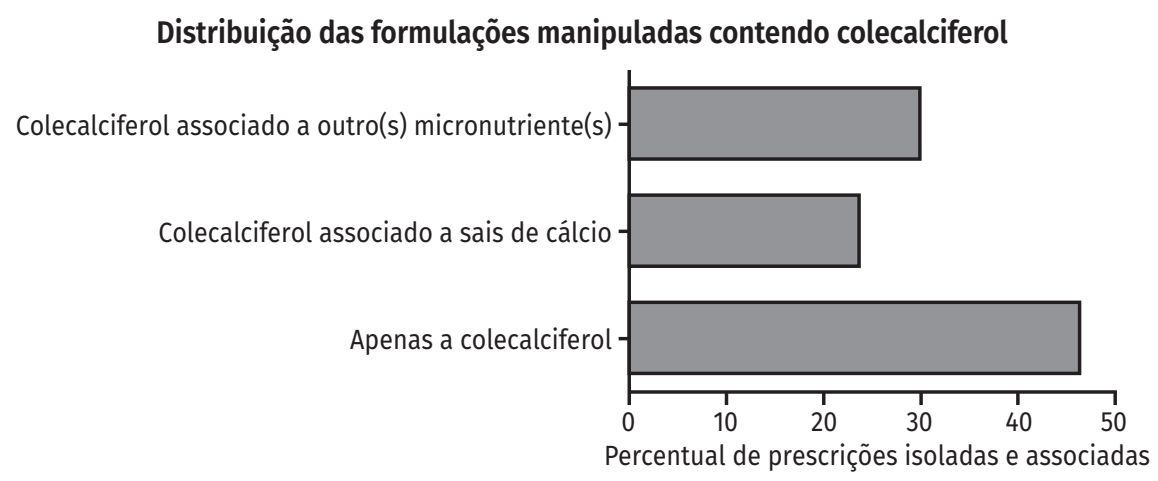

Figura 4. Distribuição das formulações manipuladas contendo colecalciferol.b

Adicionalmente, a hipercalcemia crônica pode levar à formação de cálculos e nefrocalcinose $(17,39)$. Relatos de casos discutindo quadros como este, têm sido cada vez mais descritos na literatura, sobretudo, em decorrência de uma conjunção de fatores como: a automedicação estimulada por uma legislação que permite o fácil acesso da população a suplementos vitamínicos, a prescrição de altas doses dessa vitamina por profissionais de saúde sem antes se estabelecer um diagnóstico preciso, além da manipulação deste insumo quando em desacordo as BPM $(9,14-20)$.

\section{Distribuição das prescrições magistrais contendo colecalciferol por profissionais de saúde}

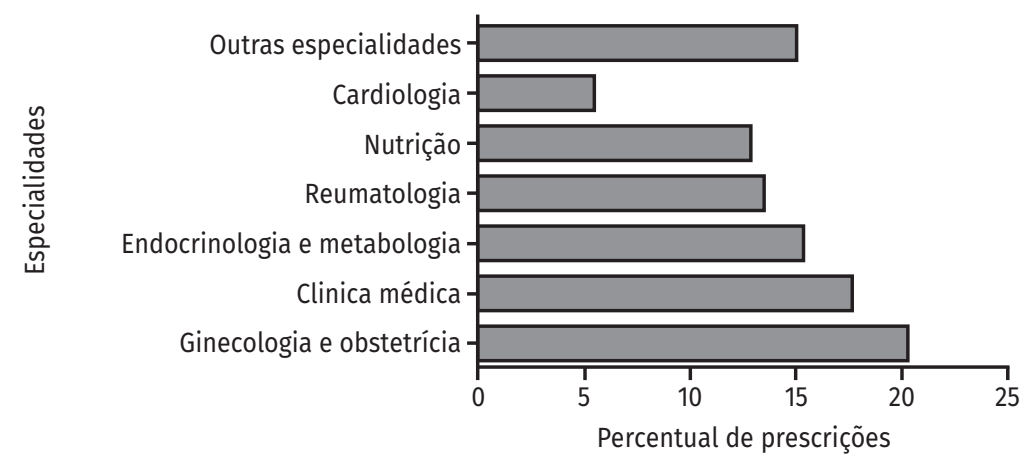

Figura 5. Distribuição das prescrições magistrais contendo colecalciferol por profissionais de saúde habilitados.

Além do exposto, é preciso considerar que colecalciferol nem sempre é administrado isoladamente, mas também em associação com um ou mais sais de cálcio e/ou a outros micronutrientes, como pôde ser constatado nas 69 diferentes apresentações comerciais expostas na Tabela 1. No nosso estudo, dentre as 896 prescrições analisadas, pouco mais da metade apresentavam-se em associação como pode ser observado no gráfico da Figura 4.

A razão para administração de colecalciferol associada a um ou mais sais de cálcio ou a outros micronutrientes como fósforo, deve-se ao aumento da absorção destes minerais no intestino estimu- lados pela vitamina $\mathrm{D}$. Sem vitamina $\mathrm{D}$, apenas $10-15 \%$ do cálcio e $60 \%$ do fósforo da dieta são absorvidos. Em quantidade suficiente, a vitamina D aumenta em 30-40\% a absorção do cálcio e em $80 \%$ a do fósforo $(3,5)$. Assim, preparações com vitamina $\mathrm{D}$ que contenham cálcio e/ou fósforo em doses elevadas podem elevar o risco de hipercalcemia e hiperfosfatemia, respectivamente (13).

Além de interações com outras vitaminas e minerais, colecalciferol também interage com fármacos de outras classes terapêuticas, como os diuréticos tiazídicos, aumentando o risco de hipercalcemia (13). Este é um tipo de interação perfei- 
tamente possível e com grandes chances de ocorrência visto que, pacientes idosos apresentam risco aumentado de deficiência de vitamina $\mathrm{D}$, fazendo o uso da suplementação, e não raro apresentam comorbidades como hipertensão arterial e insuficiência renal $(2,40)$. Diante disto, o auxílio do farmacêutico na gestão farmacoterapêutica desse paciente faz-se de extrema importância em qualquer âmbito, seja hospitalar ou na farmácia comunitária, e pode evitar várias interações medicamentosas e efeitos adversos contribuindo com o sucesso terapêutico do paciente de um modo geral (41).

É importante salientar que as recomendações tanto pelo farmacêutico quanto por qualquer outro profissional de saúde habilitado transcendem as terapias exclusivamente medicamentosas. Dado o fármaco aqui discutido, as principais causas da prevalência relacionada a seus baixos níveis séricos na população, estão ligadas a hábitos e comportamentos comuns ao estilo de vida moderno dentre eles o sedentarismo, baixa exposição à luz solar e alimentação desequilibrada. Orientações voltadas a hábitos de vida saudáveis, como atividade física regular, exposição solar moderada e alimentação equilibrada ainda parecem ser a forma mais efetiva de promover saúde e prevenir doenças, sejam elas relacionadas ou não à hipovitaminose D (5).

\section{CONCLUSÃO}

Condições clínicas envolvendo intoxicação por vitamina $\mathrm{D}$ têm sido cada vez mais relatadas na literatura, em decorrência de um conjunto de fatores como a automedicação estimulada por uma legislação que possibilita o fácil acesso da popula- ção a suplementos vitamínicos; a prescrição de altas doses dessa vitamina por profissionais de saúde sem antes se estabelecer um diagnóstico preciso e a manipulação deste insumo quando em desacordo com as BPM.

O doseamento da concentração sérica de 25(OH)D3 é a ferramenta mais adequada na avaliação da carga corpórea para o estabelecimento de um regime terapêutico seguro numa base individual. A farmácia magistral contribui de forma relevante neste aspecto, uma vez em que foge das dosagens 'genéricas' produzidas pela indústria, possibilitando tratamentos personalizados, seguros e de qualidade, dentro de uma conduta de BPM. Não obstante, a presença constante do profissional farmacêutico, neste estabelecimento possibilita a orientação adequada do paciente tanto em relação a sua terapia medicamentosa quanto a não-medicamentosa.

Por fim, a execução da farmacovigilância em relação ao uso do colecalciferol tem sido um desafio, sobretudo, pela dificuldade em identificar os sintomas clínicos associados à sua utilização. Além disso, a necessidade de um acompanhamento farmacoterapêutico mais efetivo, como o monitoramento da resposta terapêutica juntamente ao prescritor, deve ser tratada como uma perspectiva a ser atingida.

\section{AGRADECIMENTOS}

Os autores agradecem à Farmácia Escola Carlos Drummond de Andrade (FECDA) e ao Núcleo de Desenvolvimento Farmacêutico e Cosmético (NUDFAC), ambos situados na Universidade Federal de Pernambuco (UFPE).

\section{REFERÊNCIAS}

1. Unger MD. Determinação dos níveis de vitamina D em uma amostra de indivíduos saudáveis da população brasileira. [Tese]. São Paulo: Universidade de São Paulo; 2009.

2. Pinheiro TM. A Importância Clínica da Vitamina D. [Dissertação]. Porto: Universidade Fernando Pessoa. 2015.

3. Holick MF. Vitamin D Deficiency. N Engl J Med. 2007; 357: 266-81. DOI: 10.1056/NEJMra070553.
4. Fraser WD, Milan AM. Vitamin D Assays: Past and Present Debates, Difficulties, and Developments. Calcif Tissue Int. 2013; 92 (2): 118-127. DOI: 10.1007/s00223012-9693-3.

5. Lichtenstein A, Ferreira-Junior M, Sales MM, Aguiar FB, Fonseca LAM, Sumita NM, Duarte AJS. Vitamina D: ações extraósseas e uso racional. Rev. Assoc. Med. Bras. 2013; 59 (5): 495-506. DOI: 10.1016/j. ramb.2013.05.002. 
6. Pesarini JR. Níveis séricos de vitamina D associados com indicadores da síndrome metabólica na população brasileira. [Dissertação]. Rio Claro: Universidade Estadual Paulista; 2013.

7. Wimalawansa SJ. Vitamin D in the New Millennium. Curr Osteoporosis Rep.2012; 10(1):4-15. DOI: 10.1007/ s11914-011-0094-8.

8. Alves M, Bastos M, Leitão F, Marques G, Ribeiro G, Carrilho F. Vitamina D - importância da avaliação laboratorial. Rev Port Endocrinol Diabetes Metab. 2013; 8, (1): 32-39. DOI: 10.1016/j.rpedm.2012.12.001.

9. Marins TA, Galvão TFG, Korkes F, Malerbi DAC, Ganc AJ, Korn D, Wagner J, Guerra, JCC, Borges-Filho WM, Ferracini FT, et al. Intoxicação por vitamina D: relato de caso. Einstein. 2014; 12(2): 242-244. DOI: 10.1590/ S1679-45082014RC2860.

10. IOM. Institute of medicine. Dietary reference intakes for calcium and vitamin D. Washington (DC): National Academies Press; 2011.

11. Paula FJ, Rosen CJ. Vitamin D safety and requirements. Arch Biochem Biophys. 2012; 523(1):64-72. DOI: 10.1016/j.abb.2011.12.002.

12. Autier P, Boniol M, Pizot C, Mullie P. Vitamin D status and ill health: a systematic review. Lancet Diabetes Endocrinol. 2014; 2(1): 76-89. DOI: 10.1016/S22138587(13)70165-7.

13. Hathcock JN, Shao A, Vieth R, Heaney R. Risk assessment for vitamin D. Am J Clin Nutr. 2007; 85(1):6-18.

14. Gallo E,Giocaliere E, Pugi A, Menniti-Ippolito F, Bettoni D, Capurro F, Pieraccine G, Moneti G, Mugelli A, Firenzuoli F, et al. Lack of standardization in a cod liver oil dietary supplement: Three cases of hypervitaminosis D. Eur J Integr Med 2. 2010; 217-265. DOI: 10.1016/j. eujim.2010.09.176.

15. Jacobsen RB, Hornek BW, Schimidt GA, Schilling ML. Hypervitaminosis D Associated with a Vitamin D Dispensing Error. Ann Pharmacother. 2011; 45(10): 52. DOI: 10.1345/aph.1Q330.

16. Lorencio FG, Rubio E, Navarro IB, Sacristán BP, Rodríguez RP, López FJG. Hypercalcemia, hypervitaminosis A and 3-epi-25-OH-D3 levels after consumption of an "over the counter" vitamin D remedy. A case report. Food Chem Toxicol. 2012; 50(6): 2106-2108. DOI: 10.1016/j.fct.2012.03.001.

17. Guerra V, Neto OMV, Laurindo AF, De Paula FJ, Moysés Neto M. Hipercalcemia e prejuízo de função renal associados à intoxicação por vitamina $\mathrm{D}$ : relato de caso. J Bras Nefrol. 2016; 38(4):466-469 DOI: 10.5935/01012800.20160074.

18. Sath S, Shah S, Rafiq SN, Jeelani I. Hypervitaminosis D in Kashmiri Population: A Case Series of 11 Patients. Int. J. Med. Sci. 2016; 3(2):1-6 DOI: 10.14445/23939117/ IJMS-V3I2P10.
19. Araki T, Holick MF, Alfonso BD, Charlap E, Romero CM, Rizk D, Newman, LG. Vitamin D intoxication with severe hypercalcemia due to manufacturing and labeling errors of two dietary supplements made in the United States. J Clin Endocrinol Metab. 2011;96 (12):36033608. DOI: $10.1210 /$ jc. 2011-1443.

20. Bell DA, Crooke MJ, Hay N, Glendenning P. Prolonged vitamin D intoxication: presentation, pathogenesis and progress. Intern Med J. 2013; 43(10):1148-1150. DOI: 10.1111/imj.12269.

21. BRASIL. Agência Nacional de Vigilância Sanitária. Resolução RDC nº 67, de 8 de outubro de 2007. Dispõe sobre Boas Práticas de Manipulação de Preparações Magistrais e Oficinais para Uso Humano em farmácias. Diário Oficial da União, no 132, 11 de julho de 2007. Seção 2, p. 29-58.

22. BRASIL. Agência Nacional de Vigilância Sanitária. Resolução RDC nº 87, de 21 de novembro de 2008. Altera o Regulamento Técnico sobre as Boas Práticas de Manipulação em Farmácias. Diário Oficial da União, n 228, 24 de novembro de 2008, Seção 1, p. 58-9.

23. Ferreira AO. Guia Prático da Farmácia Magistral. 3.ed. Juiz de Fora, 2008.

24. Penm J, Chaar B, Rose G, Moles R. Pharmacists' influences on prescribing: validating a clinical pharmacy services survey in the Western Pacific Region. Res Social Adm Pharm. 2015;11(1):63-73. DOI: 10.1016/j.sapharm.2014.04.001.

25. Bueno MM. Implantação, evolução, aspectos técnicos e perspectivas da regulamentação técnica de biodisponibilidade relativa e bioequivalência de medicamentos genéricos e similares no Brasil [dissertação]. São Paulo: Universidade de São Paulo; 2005.

26. Aulton ME. Delineamento de formas farmacêuticas. 2. ed. Porto Alegre: Artmed; 2005.

27. Maeda SS, Borba VZC, Camargo MBR, Silva DMW, Borges JLC, Bandeira, Lazaretti-Castro, M. Recomendações da Sociedade Brasileira de Endocrinologia e Metabologia para o diagnóstico e tratamento da hipovitaminose D. Arq Bras Endocrinol Metab. 2014; 58/5. DOI: 10.1590/0004-2730000003388.

28. Stokes CS, Lammert F. Vitamin D supplementation: less controversy, more guidance needed. 2016. DOI: 10.12688/f1000research.8863.1.

29. Dimitris MC, Perumal N, Craig-Barnes HA, Leadley M, Mahmud AA, Baqui AH, Roth DE. Effect of weekly high-dose vitamin D3 supplementation on serum cholecalciferol concentrations in pregnant women. J Steroid Biochem Mol Biol. 2016;158:76-81. DOI: 10.1016/j.jsbmb.2016.01.007.

30. Jetter A, Egli A, Dawson-Hughes B, Staehelin HB, Stoecklin E, Goessl R, Henschkowski J, Bischoff-Ferrari HA. Pharmacokinetics of oral vitamin D3 and calcifediol. Bone. 2014; 59:14-19. DOI: 10.1016/j. bone.2013.10.014. 
31. Mistretta VI, Delanaye P, Chapelle JP, Souberbielle JC, Cavalier E. Vitamin D2 or vitamin D3? Rev Med Internet.2008; 29(10):815-820. DOI: 10.1016/j.revmed.2008.03.003.

32. Remondi FA, Oda S, Cabrera MAS. Não adesão à terapia medicamentosa: da teoria a prática clínica. Rev Ciênc Farm Básica Apl. 2014; 35(2):177-185.

33. CCG. Clinical commissioning group. Vitamin D Clinical Guideline. Cumbria, 2013.

34. WHO. Guideline: Vitamin D supplementation in pregnant women. Geneva, World Health Organization, 2012.

35. The endocrine society. Evaluation, Treatment, and Prevention of Vitamin D Deficiency: An Endocrine Society Clinical Practice Guideline. J Clin Endocrinol Metab. 2011; 96(7): 1911-1930.

36. Zittermann A, Ernst JB, Gummert JF, Börgermann J. Vitamin D supplementation, body weight and human serum 25-hydroxyvitamin D response: a systematic review. Eur J Nutr. 2014; 53(2):367-374. DOI: 10.1007/s00394-0130634-3.
37. Lhamo Y, Chugh PK, Tripathi CD. Vitamin D Supplements in the Indian Market. Indian J Pharm Sci. 2016; 78(1):41-47.

38. Sharma LK, Dutta D, Sharma N, Gadpayle AK. The increasing problem of subclinical and overt hypervitaminosis D in India: An institutional experience and review. Nutrition. 2017;34:76-81. DOI: 10.1016/j. nut.2016.09.014.

39. Moysés-Neto M, Guimarães FM, Ayoub FH, Vieira-Neto OM, Costa JA, Dantas M. Acute renal failure and hypercalcemia. Ren Fail. 2006;28(2):153-159.

40. Zaslavsky C, Gus I. Idoso: Doença Cardíaca e Comorbidades. Arq Bras Cardiol. 2002(79):635-639. DOI: 10.1590/S0066-782X2002001500011.

41. Andrade MA, Silva MVS, Freitas O. Assistência Farmacêutica como Estratégia para o Uso Racional de Medicamentos em Idosos. Semina: Ciênc Bio e da Saúde. 2004; 25(1). DOI: 10.5433/1679-0367.2004v25n1p55. 